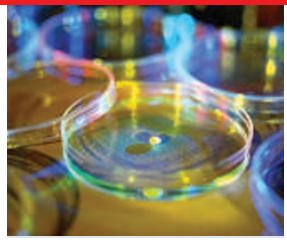

TB DIAGNOSIS CHANGE CAUSES CONFUSION Identifying drug-resistant tuberculosis is a global problem.

www.nature.com/news

\title{
Fly library boosts gene tool supply
}

Researchers have developed a library of transgenic fruitflies that will allow them to investigate genes in specific tissues or developmental stages across the whole of the insect's genome. The library, which consists of genes known as RNA interference (RNAi) transgenes, is described this week in Nature (see page 151). The transgenes act to silence their target genes, enabling researchers to work out the function of genes that would be lethal if switched off throughout the whole organism.

The fruitfly Drosophila is a model organism for developmental biologists. And Drosophila researchers have been using RNAi for years, but typically for testing only a few genes at a time. "We didn't invent anything here," says Barry Dickson, a molecular biologist at the Research Institute of Molecular Pathology in Vienna and lead author of the latest paper. "We were just crazy enough to go for the whole genome." Previously, genome-wide screens were used only in cell-culture experiments, and results had to be confirmed in live flies.

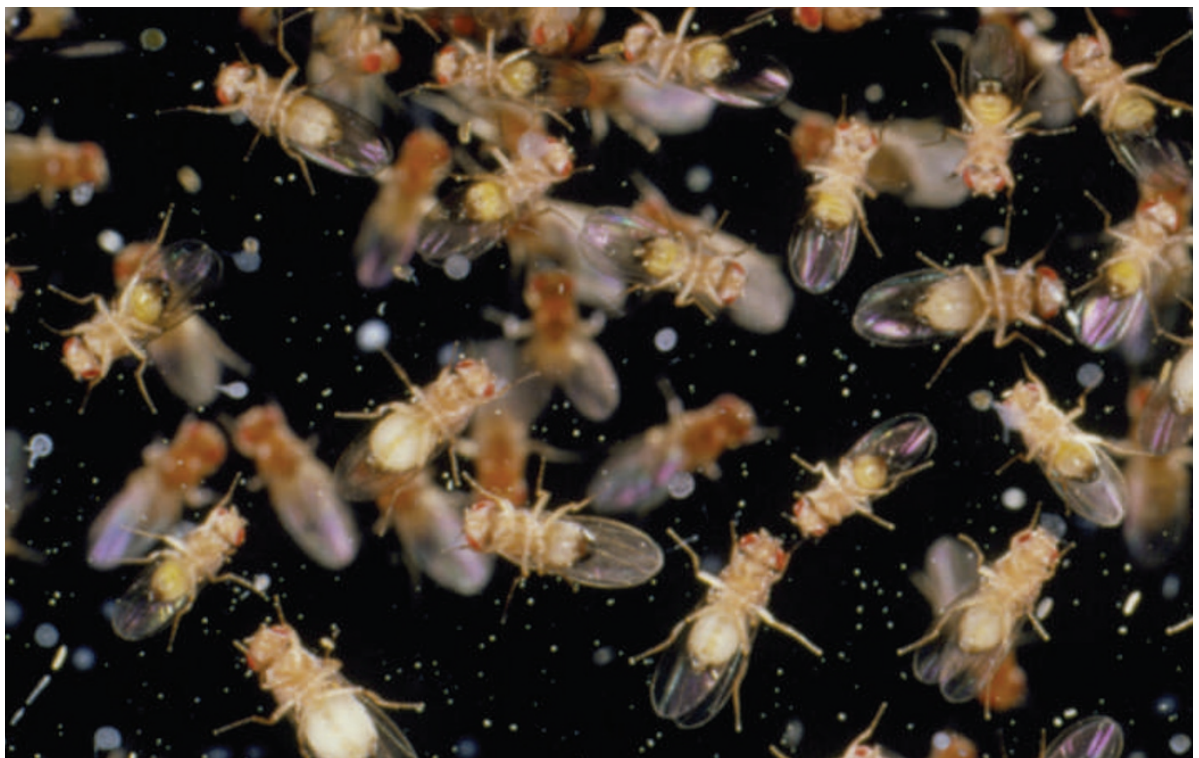

Tiny triumph: a collection of 22,270 lines of fruitfly are available for the study of developmental genes.

Now, Dickson and his colleagues have created a collection of 22,270 lines of transgenic fruitfly in which 12,088 genes can be silenced - about $88 \%$ of the fly's predicted proteincoding genes. Each fly contains an RNAi transgene that silences a targeted gene only in the presence of a protein called GAL4. Crossing the RNAi-containing strain with a strain that expresses GAL4 in a specific tissue activates RNAi only in that tissue. In Drosophila, unlike in many other insects, the RNAi signal does not travel from cell to cell.

The lines offer researchers the chance to trawl for tissuespecific functions of genes involved in fruitfly development. Dickson's lab, for

involved in eye development, courtship behaviour and the RNAi mechanism itself.

"This library is a great, great tool," says Michael Boutros, a geneticist at the German Cancer Research Center in Heidelberg. "I think it will be heavily used in the future." Boutros's lab plans to use the strains to study the Drosophila immune system.

The library generated by Dickson's group is available through the Vienna Drosophila RNAi Center, which opened in April. It already sends out about 2,000 strains each week. Potential users would do well to order soon, though: because the centre doesn't have the funds to maintain duplicate lines, there is no back-up when a vial of flies dies. The facility is curexample, studies a gene called fruitless that regulates mating behaviour. The gene's role went undiscovered for years because flies lacking it don't survive. But the auspicious discovery of fly mutants in which one form of the fruitless protein was absent in a select group of neurons revealed that the gene was crucial in triggering courtship behaviour in male flies.

In their latest study, Dickson's team took a sample of 50 genes that were lethal when silenced in all the fly's cells, and silenced them selectively in the eye, wing or thorax. Only a third of the silenced genes were still lethal. Another third were found to cause specific developmental defects in the tissues in which they were expressed. Researchers have already started to use the lines to search for genes
Other Drosophila RNAi libraries are also in the works. One, created by Ryu Ueda of the National Institute of Genetics in Shizuoka,
Japan, contains 13,000 strains so far. "This is useful because the two projects together give us more strength to cover the full genome," says Christophe Antoniewski of the Pasteur Institute in Paris. Antoniewski has been using Ueda's lines to study how RNA regulates gene expression, and plans to order lines from Dickson as well.

A third library, still in early development, is being created at Norbert Perrimon's lab at Harvard Medical School in Boston. Whereas Dickson and Ueda created their libraries by randomly inserting the RNAi transgenes, Perrimon aims to target his transgenes to a specific region in the genome. This ensures that a transgene will not land in a region of the genome that may affect the gene's expression. "The level of expression is critical," says Perrimon, "and expression is very position-specific." RNAi transgenes that aren't fully expressed can lead to false-negative results.

Meanwhile, Dickson's group has found that low expression of an RNAi transgene can often be counteracted by overexpressing a gene called dicer-2 that is involved in the RNAi pathway. "This usually helps, but it can also be dangerous," says Dickson. Overexpressing dicer-2 can increase the risk of inadvertently silencing other genes.

Such caveats are always present with highthroughput procedures, says Desplan. "Until we see how people use it, there's a wait and see position," he says. "But I'm very optimistic." -

Heidi Ledford 